\title{
Multi-layer MoS2 : An Effective Barrier Enhancer and a Promising Nanofiller for Metal Protection
}

\section{Jing Wang}

Institute of oceanology, Chinese academy of sciences

Ning Wang ( $\nabla$ wangning@qdio.ac.cn )

Institute of oceanology, Chinese academy of sciences

Qianyu Zhao

Institute of oceanology, Chinese academy of sciences

Chengyue Ge

Institute of oceanology, Chinese academy of sciences

\section{Baorong Hou}

Institute of oceanology, Chinese academy of sciences

Guichang Liu

Dalian University of Technology

\section{Wen Sun}

Dalian University of Technology

\section{Yiteng Hu}

Institute of oceanology, Chinese academy of sciences

\section{Yanli Ning}

Institute of oceanology, Chinese academy of sciences

\section{Research Article}

Keywords: Molybdenum disulfide, Nanosheet, Composite coating, Barrier properties, Metal protection

Posted Date: September 16th, 2021

DOI: https://doi.org/10.21203/rs.3.rs-879751/v1

License: (c) (i) This work is licensed under a Creative Commons Attribution 4.0 International License.

Read Full License 


\section{Abstract}

Two-dimensional nanomaterials are of great interest because of their unique properties and the great application potential in various fields. In this study, the performance of few-layer $\mathrm{MoS}_{2}$ nanosheets (MDNSs) as new nanofillers to enhance the barrier properties of poly(vinyl butyral) (PVB) is investigated. Gas permeability tests show that well-dispersed MDNSs can prevent at least $98 \%$ of oxygen molecules and $31 \%$ of water vapour from penetrating through the matrix of MDNS/PVB composites.

Electrochemical analyses reveal that the corrosion rate of brass coated with the resulting composite coating is as low as $1.35 \times 10^{-8} \mathrm{~mm} /$ year, which is six orders of magnitude smaller than that of brass protected by a pristine PVB coating. These results indicate that MDNSs are highly effective barrier enhancers and suitable candidate materials for metal protection.

\section{Introduction}

In recent years, the discovery of the extraordinary properties of graphene has greatly promoted advances in the study of two-dimensional materials (2D materials). Compared with their bulk counterparts, atomically thin $2 \mathrm{D}$ materials have a wide range of unique properties ${ }^{[1-6]}$. It has been reported that $2 \mathrm{D}$ materials hold great promise for a variety of applications such as optoelectronics ${ }^{[7-10]}$, photocatalysts $[11,12]$, photovoltaic devices ${ }^{[13,14]}$, supercapacitors ${ }^{[15]}$, fuel cells ${ }^{[16,17]}$, batteries ${ }^{[18-20]}$, sensors $[21,22]$, and so on ${ }^{[23]}$. To date, 2D materials have been reported to come in a wide range of types, such as hexagonal boron nitride ( $h$-BN) ${ }^{[24-27]}$, transition metal dichalcogenides (TMDs, such as $\mathrm{MoS}_{2}$ and $\mathrm{WS}_{2}$ ) $[28,29]$, layered metal oxides (such as $\mathrm{MoO}_{3}$ and $\mathrm{LaNb}_{2} \mathrm{O}_{7}$ ) ${ }^{[30-32]}$, layered double hydroxides (LDHs, such as $\left.\mathrm{Mg}_{6} \mathrm{Al}_{2}(\mathrm{OH})_{16}\right)^{[33,34]}$, metal halides (such as $\mathrm{Pbl}_{2}$ and $\mathrm{MgBr}_{2}$ ) ${ }^{[35]}$ and other layered materials ${ }^{[36,37]}$.

Among all discovered 2D materials, monolayer and multi-layer TMDs continue to attract tremendous research interest because the most are semiconductors with sizable bandgaps; furthermore, some are naturally abundant. $\mathrm{MoS}_{2}$ has received considerable attention in the TMD family because of its unique physical, optical and electrical properties ${ }^{[38]}$. For example, monolayer $\mathrm{MoS}_{2}$ is a non-centrosymmetric material with a direct bandgap, exhibiting interesting phenomena such as strong photoluminescence, a high current on/off ratio in field-effect transistors, and efficient valley and spin control by optical helicity [39-41]. In addition, monolayer and multi-layer $\mathrm{MoS}_{2}$ nanosheets (MDNSs) also possess some attractive properties, such as high mechanical strength (30 times stronger than steel) ${ }^{[42]}$, good heat-insulating properties (the thermal conductivity of MDNSs is $0.40-1.59 \mathrm{~W} / \mathrm{mK}$ ), and so on ${ }^{[43-45]}$.

To harness the exotic properties of MDNSs for applications, a simple route is to incorporate them in various kinds of functional materials, especially in polymer materials. To date, the synthesis of MDNS/polymer hybrid materials has attracted great interest because, compared with pristine polymers, they have significantly improved thermal stability, fire resistance, along with mechanical and electrical properties. As analogues of graphene, it is believed that MDNSs are also hardly permeable to gases and 
water vapour ${ }^{[46-48]}$. Dispersing MDNSs in polymer matrices may improve their qualities as a physical barrier because MDNSs with ultrahigh aspect ratios can significantly increase the tortuosity of diffusion pathways of molecules in polymer matrices at extremely low loadings. As barrier property enhancers, MDNSs could be excellent nanofillers for metal protection because they may increase the corrosion resistance and extend the lifetime of polymer coatings. In addition, MDNSs can also endow polymer coatings with some unique characteristics that are desirable for engineering applications, such as enhanced mechanical properties, advanced abrasion performance, and improved thermal resistance. However, to the best of our knowledge, there have been no reports describing MDNSs as barrier enhancers for metal protection until now. In this work, multi-layer MDNSs were first peeled from commercial $\mathrm{MoS}_{2}$ by liquid-exfoliation technology and then incorporated into poly(vinyl butyral) (PVB), a priming paint material used for metal protection. Compared with pristine PVB, MDNS/PVB nanocomposites (MDNS/PVBs) exhibit remarkably enhanced oxygen and moisture barrier properties and can effectively prevent metal substrate corrosion for a much more extended period.

\section{Methods}

\subsection{Synthesis of liquid-exfoliated $\mathrm{MoS}_{2}$}

The MDNSs were prepared using a liquid-phase exfoliation technique similar to that reported by Jonathan et al. Typically, commercial $\mathrm{MoS}_{2}$ powders (Aladdin, Shanghai) were exfoliated in NVP with the

assistance of sonication ${ }^{[3]}$. Typically, $0.2 \mathrm{~g} \mathrm{MoS}_{2}$ powder was exfoliated in $20 \mathrm{~mL} \mathrm{NVP}$ with $120 \mathrm{~min}$ of ultrasonic treatment. The obtained suspension was first left standing for $1 \mathrm{~h}$ at room temperature. To remove large $\mathrm{MoS}_{2}$ particles, the upper dispersion was subsequently centrifuged at $500 \mathrm{rpm}$ for $60 \mathrm{~min}$. Then, the resultant dispersion was centrifuged at 10,000 rpm for $120 \mathrm{~min}$ to collect MDNSs. The supernatant was decanted, and the residue was alternately washed with deionized water and ethanol more than 3 times ( $45 \mathrm{~mL}$ each time). The obtained solid was finally dried at room temperature under vacuum.

\subsection{Preparation of MDNS/PVB composite coatings}

MDNSs/PVBs with different mass fractions of $\operatorname{MDNSs}(0,0.1,0.2,0.3,0.4,0.5$ and 1.0 wt.\%) were prepared by the following method. First, some MDNSs were dispersed in $20 \mathrm{~mL}$ methanol with continuous stirring and sonication for $24 \mathrm{~h}$. Then, $2 \mathrm{~g}$ PVB powder was added to the dispersion under stirring for one day, yielding a viscous solution with uniformly dispersed MDNSs and no settling (the viscous solution is denoted as MDNS/PVB paint). Before coating, a brass tablet ( $\Phi 20 \mathrm{~mm} \times 2 \mathrm{~mm})$, which was embedded in a $29 \mathrm{~mm} \times 29 \mathrm{~mm} \times 11 \mathrm{~mm}$ epoxy matrix, was first polished with $\mathrm{SiC}-1,000$ paper and then washed with acetone. After being air blow-dried, the brass tablet, which had an exposed surface of $3.14 \mathrm{~cm}^{2}$, was coated by MDNS/PVB coatings via a dip-coating method. Typically, the brass tablet was first immersed in the MDNS/PVB paint for $30 \mathrm{~s}$. Then, the brass tablet was slowly withdrawn from the dispersion. Subsequently, the paint-covered brass tablet was placed horizontally in the air at 20 
${ }^{\circ} \mathrm{C}$ for $24 \mathrm{~h}$ to give a final coating with a thickness in the range of $52.4 \pm 3.0 \mu \mathrm{m}$. The obtained specimens were denoted as MDNSs/PVB ${ }_{0}, \mathrm{MDNSs}_{\mathrm{PVVB}} \mathrm{PV}_{0.1}, \mathrm{MDNSs}_{\mathrm{PV}} \mathrm{PV}_{0.2}, \mathrm{MDNSs} / \mathrm{PVB}_{0.3}, \mathrm{MDNSs} / \mathrm{PVB}_{0.4}$, MDNSs/PVB 0.5 and $M D N S s / P B_{1.0}$.

\subsection{Electrochemical tests}

The electrochemical measurements were carried out in a conventional three-electrode electrochemical cell employing $3.5 \mathrm{wt}$ \% $\mathrm{NaCl}$ aqueous solution as the electrolyte. A saturated calomel electrode (SCE) was used as the reference electrode, and a platinum electrode was employed as the counter electrode. The working electrode was a coated brass sample. Both electrochemical impedance spectroscopy (EIS) and Tafel polarization were performed on a CHI660B electrochemical workstation (Chenhua, Shanghai). The EIS measurements were carried out at open circuit potential $\left(E_{\mathrm{OCP}}\right)$ with applied sinusoidal perturbations of 50 (for intact coatings) and $5 \mathrm{mV}$ (for defective coatings) over a frequency range of $0.01 \mathrm{~Hz}-100 \mathrm{kHz}$. The Tafel polarization was scanned from the cathodic to the anodic direction (from $E_{\mathrm{OCP}}-300 \mathrm{mV}$ to $E_{\mathrm{OCP}}+300 \mathrm{mV}$ ) with a scan rate of $1 \mathrm{mV} / \mathrm{s}$.

\subsection{Apparatus and Characterization}

The MDNSs were characterized by scanning electron microscopy (SEM, JEOL-5600LV, Japan), transmission electron microscopy (TEM, FEI Tecnai F30, Holland), and atomic force microscopy (AFM, Dimension 3100, Veeco, CA). Coating thickness was measured by a portable thickness gauge (TR110, Beijing Time High Technology Ltd., China). The water vapour transmission rate (WVTR) experiment was performed under ASTM F1249 using a water vapour permeability analyser (Permatran-W 3/33TO, MOCON, America) at $25^{\circ} \mathrm{C}$ and $100 \% \mathrm{RH}$. The oxygen transmission rate (OTR) of the coatings was tested by ASTM D-3985 using an oxygen permeation meter (Ox-Tran 2/61, MOCON, America) at $25^{\circ} \mathrm{C}$ and $40 \% \mathrm{RH}$. The morphologies of the brass substrates were observed by SEM and metallographic microscopy (BX51M, Olympus, Japan). Corrosion products on the brass substrates were analysed by Raman spectroscopy (DXR, Thermo Fisher Scientific, America).

\section{Results And Discussion}

Bulk $\mathrm{MoS}_{2}$ has a layered structure similar to that of graphite and is built up from monolayer $\mathrm{MoS}_{2}$, which consists of a single layer of molybdenum atoms sandwiched between two layers of sulfur atoms in a trigonal prismatic structure. As the $\mathrm{MoS}_{2}$ monolayers in bulk MoS are weakly bonded by van der Waals interactions ${ }^{[49]}$, monolayer and multi-layer MDNSs can be peeled from bulk $\mathrm{MoS}_{2}$ through chemical, liquid, or mechanical exfoliation technologies ${ }^{[47]}$. In this work, a liquid-phase exfoliation technique was used to prepare MDNSs because they provide simple routes for the large-scale synthesis of 2D materials at room temperature ${ }^{[3,50,51]}$. Figure 1 (a) shows a photograph of suspensions containing the starting $\mathrm{MoS}_{2}$ powders and the as-prepared MDNSs in N-vinyl-pyrrolidinone (NVP). The suspension of starting $\mathrm{MoS}_{2}$ powders in NVP is silver-grey, and it becomes transparent and shows evident precipitation even after a short period of storage. After liquid exfoliation and extensive purification, a yellow-green 
dispersion of $\mathrm{MoS}_{2}$ nanomaterials was obtained. The suspension contains a high concentration of MDNSs. It is highly stable and shows no precipitation after being stored for one week. SEM images reveal that the starting $\mathrm{MoS}_{2}$ powders have flake-like structures with a lateral size of several micrometres, whereas the MDNSs have no specific structure due to their ultra-thin nature (Fig. (S1)). TEM investigations reveal that the MDNSs are electron transparent, and the majority of them are twodimensional nanosheets with lateral sizes of hundreds to thousands of nanometres (Fig. 1(b)). In addition, if the nanosheet is locally parallel to the electron beam at a fold, high-resolution TEM (HR-TEM) analysis of folding at the edges can give the number of layers by direct visualization. In this work, it is proposed that the five dark lines shown in the HR-TEM of a MDNS fold reveal that the prepared MDNSs are five-layer nanosheets (Fig. 1(c) ${ }^{[52]}$. To confirm the layer number of MDNSs, atomic force microscopy (AFM) analysis of the highly unfolded nanosheets was also carried out. AFM shows that the prepared MDNSs have a uniform thickness distribution, which indicates that the liquid-exfoliation process is highly efficient for obtaining few-layer nanosheets (Fig. 1(d)). The apparent thicknesses of two representative pieces of MDNSs measured by AFM are 3.51 and $3.44 \mathrm{~nm}$, respectively (Fig. 1(e)). The apparent thickness of monolayer $\mathrm{MoS}_{2}$ was previously reported to be $0.65-0.8 \mathrm{~nm}$. The layer number of the MDNSs is calculated to be approximately five, which is in good agreement with the HR-TEM analyses [53, $54]$.

The as-prepared MDNSs were uniformly dispersed in PVB with the aid of an ultrasonic bath, obtaining silver-grey paints. As shown in Fig. 2(a), MDNS/PVB paints remained unchanged in colour, and they exhibited no precipitation after storage for one week. Free-standing MDNS/PVB films were prepared for gas transmittance measurements. As shown in Fig. 2(b), even with very small loadings of MDNSs ( 0.1 wt.\%), a dense network of overlapping MDNSs was formed within the polymer matrix, forming a uniformly light silver grey film. This phenomenon indicates that the MDNS/PVB composite film can serve as an excellent barrier material for light, which implies that it may also hardly be permeable to gases, such as $\mathrm{O}_{2}$ and water vapour. The oxygen and water vapour transmittance rates (OTR and WVTR) of the blank and MDNS-reinforced PVB films were measured, and the results are presented in Fig. 3 . It is clear that the blank PVB film (MDNS/PVB ${ }_{0}$ ) possesses considerably higher OTR and WVTR values of $\sim 10^{5}$ $\mathrm{cm}^{3} / \mathrm{m}^{2} /$ day and $218.3 \mathrm{~g} / \mathrm{m}^{2} /$ day, respectively, revealing its poor barrier properties. This result is attributed to the porous nature of the MDNS/PVB ${ }_{0}$ film. Figure 2(c) reveals numerous nanosized pinholes at the cross-section of MDNSs/PVB ${ }_{0}$. The rapid evaporation of volatile methanol causes these pinholes. The pinholes can allow oxygen molecules to conveniently diffuse through the MDNS/PVB ${ }_{0}$ film. However, compared with MDNS/PVB ${ }_{0}$, significant decreases in the OTR and WVTR values for MDNS/PVB films are observed. Gas transmittance tests show that the OTR of MDNS/PVB films is $<1150 \mathrm{~cm}^{3} / \mathrm{m}^{2} /$ day, and the WVTR is $<150 \mathrm{~g} / \mathrm{m}^{2} /$ day. The barrier properties of MDNS/PVB films are enhanced because, on the one hand, by forcing the molecules to permeate through the film matrices in an extremely "tortuous pathway." MNDSs with a large aspect ratio can slow down the permeation of oxygen and water molecules through the composites; on the other hand, compared with MNDS/PVB ${ }_{0}$, the MNDS/PVB films possess denser structures (Table S1, Tafel polarization results reveal that MNDS/PVB films have lower porosity). SEM 
images of the cross-section of MDNS/PVB films reveal no pinholes in the matrix of PVB (Fig. 2(d-i)). The pinholes are eliminated because MDNSs can prolong the diffusion pathway of solvent molecules in the matrix of PVB, which can remarkably slow the evaporation of the solvent. Therefore, it is concluded that MBNSs are excellent barrier enhancers in reducing the gas and water vapour transmittance rates of PVB films.

To study the corrosion protection performance of the MDNS/PVB composites, the as-prepared paints were coated on brass substrates through dip-coating. The prepared samples were immersed in $3.5 \mathrm{wt} . \%$ $\mathrm{NaCl}$ solution and Tafel polarization data were collected after 4 months of immersion in $3.5 \mathrm{wt} . \% \mathrm{NaCl}$ aqueous solution (Fig. $4(\mathrm{a})$ ). The corrosion potential $\left(E_{\text {corr }}\right)$ shifted gradually to a more positive value, and the polarization current density tended to decrease when more MDNSs were incorporated into the PVB coatings. Through Tafel fitting, the Tafel polarization data can provide important information on the kinetics of corrosion reactions occurring at the metal/coating interface. According to the analysis of the polarization data, the corrosion current density $\left(I_{\text {corr }}\right)$ of different samples can be calculated using the Stern-Geary equation:

$$
I_{\text {corr }}=\frac{b_{\mathrm{a}} \times b_{\mathrm{c}}}{2.303\left(b_{\mathrm{a}}+b_{\mathrm{c}}\right)} \cdot \frac{1}{R_{\mathrm{p}}}
$$

Cathodic and anodic Tafel slopes ( $b_{c}$ and $b_{a}$, respectively) were fitted from the Tafel regions (the overpotential is more than $\pm 100 \mathrm{mV}$ ) of the cathodic and anodic branches of the polarization curves, respectively. The linear polarization resistance $\left(R_{\mathrm{p}}\right)$ was calculated from the linear regions (the overpotential was less than $\pm 20 \mathrm{mV}$ ) of the polarization curves. In addition, the corrosion rate $R_{\text {corr }}$ $(\mathrm{mm} /$ year) is calculated by:

$$
R_{\text {corr }}=\frac{k M_{\mathrm{m}} I_{\text {corr }}}{n \rho_{\mathrm{m}}}
$$

where $k$ is a constant $(3,268.5 \mathrm{~mol} / \mathrm{A}), M_{\mathrm{m}}$ is the molecular weight of brass $(\mathrm{g} / \mathrm{mol}), n$ is the number of charge transfers, and $\rho_{\mathrm{m}}$ denotes the density of brass $\left(\mathrm{g} / \mathrm{cm}^{3}\right)$. The fitting results of the polarization curves show that the $E_{\text {corr }}$ of the MDNS/PVB 0 coating is $-0.285 \mathrm{~V}$ vs. SCE, and the $E_{\text {corr }}$ of the MDNS/PVB coatings shifts positively from -0.034 to $25.5 \mathrm{~V}$ vs. SCE with increasing MDNS incorporation in the coatings (Table S1). Furthermore, the $I_{\text {corr }}$ of MDNSs/PVB ${ }_{0}$ is $2.34 \mu \mathrm{A} / \mathrm{cm}^{2}$, while that for MDNSs/PVB ${ }_{0.1}$ decreases dramatically to $3.12 \times 10^{-4} \mu \mathrm{A} / \mathrm{cm}^{2}$. The $I_{\text {corr }}$ decreases with increasing MDNS loading. Remarkably, the $I_{\text {corr }}$ decreases to $1.15 \times 10^{-6} \mu \mathrm{A} / \mathrm{cm}^{2}$ when $1.0 \mathrm{wt}$.\% MDNSs are incorporated into the PVB coating. The $R_{\text {corr }}$ of MDNS/PVB ${ }_{0}$ is $2.75 \times 10^{-2} \mathrm{~mm} /$ year, while it decreases approximately 13,000 times when only 0.1 wt.\% MDNSs are used $\left(3.66 \times 10^{-6} \mathrm{~mm} /\right.$ year, Fig. $\left.4(\mathrm{~b})\right)$. The $R_{\text {corr }}$ of MDNS/PVB 1.0 is 
as low as $1.35 \times 10^{-8} \mathrm{~mm} /$ year. The positive shift of the $E_{\text {corr }}$ of the MDNS/PVB coatings to nobler values and the decrease in $I_{\text {corr }}$ or $R_{\text {corr }}$ demonstrate an effective protective coating on the brass surface. Electrochemical impedance spectroscopy (EIS) was further utilized to investigate the protection performance of the composite coatings. During the initial immersion period, the ElS spectra reveal that both the pristine PVB coating and MDNS/PVB composite coatings possess a coating resistance as high as $\sim 10^{10}-10^{11} \Omega \cdot \mathrm{cm}^{2}$, indicating their excellent corrosion protection performance (Fig. 2(S)). Figure 5 shows the Bode plots of the MDNS/PVB coatings after 4 months of immersion in $3.5 \mathrm{wt} . \% \mathrm{NaCl}$. The Bode modulus plots show that the modulus $|Z|$ at low frequencies increases with increasing MDNS incorporation (Fig. 5(a)). The |Z| value remains almost unchanged when incorporating more than 0.4 wt.\% MDNSs into the matrix of PVB. Furthermore, Fig. 5(b) shows that the Bode phase plot of $M D N S / P V B_{0}$ includes a peak at high frequencies and a peak located in the low-frequency region (twotime constants), attributed to the coating and corrosion response, respectively. However, only one peak can be seen from the Bode phase plot of the coating reinforced with MDNSs (one-time constant), which indicates that no or extremely slight corrosion occurs at the coating/brass interface. The inhibited corrosion suggests that little electrolyte can permeate through the MDNS/PVB coatings. Therefore, an effective barrier can be fabricated at the brass surface to completely hinder the access of the electrolyte to the brass substrates when sufficient amounts of MDNSs are incorporated into the matrix of PVB. To quantitatively compare the corrosion protection performance of MDNS/PVB coatings, the Bode plots were also fitted by the electrical models presented in Fig. 5(c). With the help of ZsimpWin software, different parameters, including $R_{\mathrm{ct}}$ (charge transfer resistance), $R_{\mathrm{sol}}$ (solution resistance), $R_{\mathrm{c}}$ (coating resistance), $C_{\mathrm{dl}}$ (double layer capacitance), and $C_{\mathrm{c}}$ (coating capacitance), can be calculated based on the EIS data. Among all the parameters, $R_{\mathrm{C}}$ is used to evaluate the corrosion protection performance of the MDNS/PVB coatings because the corrosion protection performance of a polymeric coating is generally monotonically related to its $R_{\mathrm{c}}$ value. The fitting results of $R_{\mathrm{c}}$ are shown in Fig. 5(d). The $R_{\mathrm{c}}$ of MDNS/PVB ${ }_{0}$ is $4.28 \times 10^{3} \Omega \cdot \mathrm{cm}^{2}$, while it dramatically increases to $2.22 \times 10^{8} \Omega \cdot \mathrm{cm}^{2}$ when incorporating only 0.1 wt.\% MDNSs. Furthermore, with increasing MDNS loading, the $R_{\mathrm{c}}$ of the MDNS/PVB coatings gradually increases to $\sim 5 \times 10^{10} \Omega \cdot \mathrm{cm}^{2}$ (MDNSs $>0.4 \mathrm{wt}$.\%). The EIS results reveal that the MDNS/PVB coatings can provide better protection for metals than the blank PVB coating.

To further confirm whether MDNS/PVB coatings can serve as corrosion protective coatings, we removed all the coatings and characterized the underlying brass substrates after 4 months of immersion in 3.5 $w t . \% \mathrm{NaCl}$ aqueous solution at room temperature. Figure 6 shows a photograph of a freshly prepared brass sample (without coating, exposed to air for several hours only) and all the immersed brass substrates. Before immersion, all brass samples show a characteristic brass lustre to the naked eye after being polished by 1,000 SiC papers (Fig. 6(a)). After immersion, a notable colour change is observed on the substrate surface of MDNS/PVB ${ }_{0}$ due to the formation of corrosion products (Fig. 6(b)). However, for MNDS/PVB coatings, the formation of corrosion products is related to MNDS loadings in the PVB matrix. For MNDSs/PVB ${ }_{0.1}$ and MNDSs/PVB ${ }_{0.2}$, visible corrosion spots can be observed on the brass surface (Fig. 6(c,d)). However, brass substrates protected by MNDSs/PVB ${ }_{0.3}, \mathrm{MDNSs}_{\mathrm{PV}} \mathrm{PB}_{0.4}, \mathrm{MDNSs}_{\mathrm{PV}} \mathrm{PB}_{0.5}$, and 
MDNSs/PVB ${ }_{1.0}$ maintain their metallic lustre, and no noticeable colour change was observed on their surfaces (Fig. 6(e-h)). When inspected under a metallographic microscope, the corroded region on the brass substrates exhibits a red and black appearance (Fig. 7(a-d)). Nevertheless, similar to newly polished brass, uncorroded brass substrates appeared highly reflective and yellow (Fig. 7(e-h)).

Raman spectroscopy was utilized to characterize the corrosion products further. Three Raman peaks can be observed in the Raman spectra of brass substrates beneath PVB reinforced with no more than 0.3 wt.\% MDNSs (Fig. 8). The peak at $283 \mathrm{~cm}^{-1}$ is assigned to the $\mathrm{A}_{\mathrm{g}}$ mode of $\mathrm{CuO}$, and the peaks at 326 and $615 \mathrm{~cm}^{-1}$ can be indexed to the $\mathrm{B}_{\mathrm{g}}$ modes of $\mathrm{CuO}$. The Raman spectra of the corrosion products also clearly show the Raman-active modes of $\mathrm{ZnO}$, indicating the existence of $\mathrm{ZnO}$ in the products. The peak at $438 \mathrm{~cm}^{-1}$ is attributed to the $\mathrm{E}_{2}$ (high) mode, a first-order optical mode of wurtzite $\mathrm{ZnO}{ }^{[55]}$. The peaks at 380,410 , and $575 \mathrm{~cm}^{-1}$ are attributed to the $A_{1}(T O), E_{1}(T O)$, and $A_{1}(L O)$ modes of $Z n O$, which are also ascribed to the first-order optical modes of wurtzite $\mathrm{ZnO}{ }^{[56]}$. In addition, the peaks located at 203 and 334 $\mathrm{cm}^{-1}$ are $2 \mathrm{E}_{2}$ (low) and $\mathrm{E}_{2}$ (high)- $\mathrm{E}_{2}$ (low) modes, which are second-order features of $\mathrm{ZnO}$ crystals caused by multi-phonon processes ${ }^{[57]}$. The peak located at $1,072 \mathrm{~cm}^{-1}$ is assigned to TO + LO combinations, and the peak appearing at $1,158 \mathrm{~cm}^{-1}$ is attributed to the $2 \mathrm{~A}_{1}(\mathrm{LO}), 2 \mathrm{E}_{1}(\mathrm{LO})$, and $2 \mathrm{LO}$ modes of $\mathrm{ZnO}$. Therefore, the corrosion products formed on the brass surface mainly consist of $\mathrm{CuO}$ and $\mathrm{ZnO}$. The Raman spectra of MDNSs/PVB composite coatings with more than $0.3 \mathrm{wt} . \%$ MDNS loading are similar to those of a newly prepared brass substrate and exhibit no evident Raman-active vibration modes, indicating that there are few corrosion products formed on the surface of the underlying brass substrates. These results demonstrate that the MDNS/PVB coating can effectively prevent the corrosion of brass at room temperature in $3.5 \mathrm{wt} . \% \mathrm{NaCl}$ over a long time scale ${ }^{[57-59]}$.

\section{Conclusion}

In summary, we have demonstrated the potential application of liquid-exfoliated MDNSs as nanofillers to enhance the barrier properties of polymer composites for metal protection. Compared with pristine coatings, the composite coatings exhibit a higher impermeability to oxygen and water vapour. The barrier properties of the resulting composites are enhanced because well-dispersed MDNSs with a relatively high aspect ratio form a dense network of overlapping MDNSs within the polymer matrix, which increases the tortuosity of the diffusion pathways of gas molecules and impedes them from penetrating through the polymer matrix. The enhanced barrier properties are responsible for the highly desirable corrosion protection performances of the MDNS/polymer composites. Because it is easy to obtain the MDNS precursor molybdenum disulfide, which is naturally abundant, we believe that MDNSs have great potential to be used as packaging materials and, especially, as coating materials for metal protection. Due to their analogous structures, it is reasonably believed that the TMD family will launch a new era of corrosion protection materials in the near future.

\section{Declarations}




\section{Acknowledgements}

This work was supported by the National Natural Science Foundation of China (Nos. U1706225, 42006046, and 2019YFC0312103).

\section{References}

1. Butler, S. Z. et al. Progress, challenges, and opportunities in two-dimensional materials beyond graphene., 7,, 2898-2926 (2013).

2. Coleman, J. N. et al. Two-dimensional nanosheets produced by liquid exfoliation of layered materials., 331, 568-571 (2011).

3. Nicolosi, V., Chhowalla, M., Kanatzidis, M. G., Strano, M. S. \& Coleman, J. N. Liquid exfoliation of layered materials., 340, 1226419 (2013).

4. Xu, M. S., Liang, T., Shi, M. M. \& Chen, H. Z. Graphene-like two-dimensional materials. Chem. Rev, 113, 3766-3798 (2013).

5. Tang, Q. \& Zhou, Z. Graphene-analogous low-dimensional materials. Prog. Mater. Sci, 58, 1244$1315(2013)$.

6. Zhang, X. D. \& Xie, Y. Recent advances in free-standing two-dimensional crystals with atomic thickness: design, assembly and transfer strategies. Chem. Soc. Rev, 42„8187-8199 (2013).

7. Geim, A. K. \& Novoselov, K. S. The rise of graphene. Nat. Mater, 6, 183-191 (2007).

8. Molina-Sanchez, A. \& Wirtz, L. Phonons in single-layer and few-layer MoS2 and WS2. Phys. Rev. B, 84, 155413 (2011).

9. Molina-Sanchez, A., Sangalli, D., Hummer, K., Marini, A. \& Wirtz, L. Effect of spin-orbit interaction on the optical spectra of single-layer, double-layer, and bulk MoS2. Phys. Rev. B, 88, 045412 (2013).

10. Jacobs-Gedrim, R. B. et al. Extraordinary photoresponse in two-dimensional In2Se3 nanosheets., 8, 514-521 (2014).

11. Bonaccorso, F., Sun, Z., Hasan, T. \& Ferrari, A. C. Graphene photonics and optoelectronics. Nat Photonics, 4, 611-622 (2010).

12. Wang, Q. H., Kalantar-Zadeh, K., Kis, A., Coleman, J. N. \& Strano, M. S. Electronics and optoelectronics of two-dimensional transition metal dichalcogenides. Nat. Nanotechnol, 7,,699-712 (2012).

13. Yang, J. \& Shin, H. S. Recent advances in layered transition metal dichalcogenides for hydrogen evolution reaction. J. Mater. Chem, A 2, 5979-5985 (2014).

14. Yang, L. et al. Optical properties of metal-molybdenum disulfide hybrid nanosheets and their application for enhanced photocatalytic hydrogen evolution., 8,, 6979-6985 (2014).

15. Guo, C. X. et al. Layered graphene/quantum dots for photovoltaic devices. Angew. Chem. Int. Edit, 49,, 3014-3017 (2010).

16. Xu, J. et al. A sandwich-type three-dimensional layered double hydroxide nanosheet array/graphene composite: fabrication and high supercapacitor performance. J. Mater. Chem. A, 2, 1022-1031 
(2014).

17. Hanlon, D. et al. Production of molybdenum trioxide nanosheets by liquid exfoliation and their application in high-performance supercapacitors. Chem. Mater, 26, 1751-1763 (2014).

18. Uosaki, K. et al. Boron nitride nanosheet on gold as an electrocatalyst for oxygen reduction reaction: theoretical suggestion and experimental proof. J. Am. Chem. Soc, 136, 6542-6545 (2014).

19. Gong, Y. J. et al. Boron- and nitrogen-substituted graphene nanoribbons as efficient catalysts for oxygen reduction reaction. Chem. Mater, 27,, 1181-1186 (2015).

20. Liu, M. M., Zhang, R. Z. \& Chen, W. Graphene-supported nanoelectrocatalysts for fuel cells: synthesis, properties, and applications. Chem. Rev, 114, 5117-5160 (2014).

21. Sun, P. L., Zhang, W. X., Hu, X. L., Yuan, L. X. \& Huang, Y. H. Synthesis of hierarchical MoS2 and its electrochemical performance as an anode material for lithium-ion batteries. J. Mater. Chem, A 2, 3498-3504 (2014).

22. Ding, Y. L., Wen, Y. L., Van Aken, P. A., Maier, J. \& Yu, Y. Jarosite nanosheets fabricated via roomtemperature synthesis as cathode materials for high-rate lithium ion batteries. Chem. Mater, 27, 3143-3149 (2015).

23. Keeley, G. P. et al. Electroanalytical sensing properties of pristine and functionalized multilayer graphene. Chem. Mater, 26,, 1807-1812 (2014).

24. Zhi, C. Y., Bando, Y., Tang, C. C., Kuwahara, H. \& Golberg, D. Large-scale fabrication of boron nitride nanosheets and their utilization in polymeric composites with improved thermal and mechanical properties. Adv. Mater, 21,, 2889 (2009).

25. Lee, K. H. et al. Large-scale synthesis of high-quality hexagonal boron nitride nanosheets for largearea graphene electronics. Nano Lett, 12, 714-718 (2012).

26. Shi, Y. M. et al. Synthesis of few-layer hexagonal boron nitride thin film by chemical vapor deposition. Nano Lett, 10, 4134-4139 (2010).

27. Nag, A. et al. Graphene analogues of BN: novel synthesis and properties., 4, 1539-1544 (2010).

28. Huang, X., Zeng, Z. Y. \& Zhang, H. Metal dichalcogenide nanosheets: preparation, properties and applications. Chem. Soc. Rev, 42,, 1934-1946 (2013).

29. Zeng, Z. Y. et al. Single-layer semiconducting nanosheets: high-yield preparation and device fabrication. Angew. Chem. Int. Edit, 50,, 11093-11097 (2011).

30. Chang, J. et al. Asymmetric supercapacitors based on graphene/MnO2 nanospheres and graphene/MoO3 nanosheets with high energy density. Adv. Funct. Mater, 23,, 5074-5083 (2013).

31. Tagusagawa, C., Takagaki, A., Hayashi, S. \& Domen, K. Characterization of HNbWO6 and HTaWO6 metal oxide nanosheet aggregates as solid acid catalysts. J. Phys. Chem. C, 113, 7831-7837 (2009).

32. Zhou, G. M. et al. Oxygen bridges between NiO nanosheets and graphene for improvement of lithium storage., 6, 3214-3223 (2012). 
33. Ma, R. Z., Liu, Z. P., Li, L., lyi, N. \& Sasaki, T. Exfoliating layered double hydroxides in formamide: a method to obtain positively charged nanosheets. J. Mater. Chem, 16, 3809-3813 (2006).

34. Wang, Q. \& O'Hare, D. Recent advances in the synthesis and application of layered double hydroxide (LDH) nanosheets. Chem. Rev, 112, 4124-4155 (2012).

35. Chua, C. K., Sofer, Z., Lim, C. S. \& Pumera, M. Inherent electrochemistry of layered post-transition metal halides: the unexpected effect of potential cycling of Pbl2. Chem. Eur. J, 21, 3073-3078 (2015).

36. Wang, Q. et al. High-water-content mouldable hydrogels by mixing clay and a dendritic molecular binder. Nature, 463, 339-343 (2010).

37. Zhang, X. D. et al. Single-layered graphitic-C(3)N(4) quantum dots for two-photon fluorescence imaging of cellular nucleus. Adv, Mater. 26, 4438 (2014). )

38. Chhowalla, M. et al. The chemistry of two-dimensional layered transition metal dichalcogenide nanosheets. Nat Chem, 5, 263-275 (2013).

39. Radisavljevic, B., Radenovic, A., Brivio, J., Giacometti, V. \& Kis, A. Single-layer MoS2 transistors. Nat. Nanotechnol, 6, 147-150 (2011).

40. Splendiani, A. Emerging photoluminescence in monolayer MoS2. Nano Lett, 10, 1271-1275 (2010).

41. Novoselov, K. S. et al. Two-dimensional atomic crystals. P. Natl. Acad. Sci, 102, 10451-10453 (2005).

42. Mak, K. F. et al. Tightly bound trions in monolayer MoS2. Nat. Mater, 12, 207-211 (2013).

43. Radisavljevic, B., Whitwick, M. B. \& Kis, A. Small-signal amplifier based on single-layer MoS2. Appl. Phys. Lett, 101, 043103 (2012).

44. Bertolazzi, S., Brivio, J. \& Kis, A. Stretching and breaking of ultrathin MoS2., 5, 9703-9709 (2011).

45. Liu, X. J., Zhang, G., Pei, Q. X. \& Zhang, Y. W. Phonon thermal conductivity of monolayer MoS2 sheet and nanoribbons. Appl. Phys. Lett, 103,, 133113 (2013).

46. Zhou, K. Q. et al. Preparation of poly(vinyl alcohol) nanocomposites with molybdenum disulfide (MoS2): structural characteristics and markedly enhanced properties. Rsc Adv, 2, 11695-11703 (2012).

47. Lin, B. Z., Ding, C., Xu, B. H., Chen, Z. J. \& Chen, Y. L. Preparation and characterization of polythiophene/molybdenum disulfide intercalation material. Mater. Res. Bull, 44, 719-723 (2009).

48. Bissessur, R. \& White, W. Novel alkyl substituted polyanilines/molybdenum disulfide nanocomposites. Mater. Chem. Phys, 99, 214-219 (2006).

49. Mak, K. F., He, K. L., Shan, J. \& Heinz, T. F. Control of valley polarization in monolayer MoS2 by optical helicity. Nat. Nanotechnol, 7, 494-498 (2012).

50. Varrla, E. et al. Large-scale production of size-controlled MoS2 nanosheets by shear exfoliation. Chem. Mater, 27,, 1129-1139 (2015).

51. Ding, Q. et al. Efficient photoelectrochemical hydrogen generation using heterostructures of $\mathrm{Si}$ and chemically exfoliated metallic MoS2. J. Am. Chem. Soc, 136, 8504-8507 (2014). 
52. Ahmad, I., Khan, U. \& Gun'ko, Y. K. Graphene, carbon nanotube and ionic liquid mixtures: towards new quasi-solid state electrolytes for dye sensitised solar cells. J. Mater. Chem, 21,, 16990-16996 (2011).

53. Wu, J. et al. Layer thinning and etching of mechanically exfoliated MoS2 nanosheets by thermal annealing in air. Small, 9, 3314-3319 (2013).

54. Yin, Z. Y. et al. Single-layer MoS2 phototransistors., 6, 74-80 (2012).

55. Yao, W. T. et al. Formation of uniform $\mathrm{CuO}$ nanorods by spontaneous aggregation: selective synthesis of $\mathrm{CuO}, \mathrm{Cu} 2 \mathrm{O}$, and $\mathrm{Cu}$ nanoparticles by a solid-liquid phase arc discharge process. J. Phys. Chem, B 109,, 14011-14016 (2005).

56. Dong, X. C. et al. Hybrid structure of zinc oxide nanorods and three dimensional graphene foam for supercapacitor and electrochemical sensor applications. Rsc Adv, 2, 4364-4369 (2012).

57. Han, W. J. et al. Synthesis of CdS/ZnO/graphene composite with high-efficiency photoelectrochemical activities under solar radiation. Appl. Surf. Sci, 299, 12-18 (2014).

58. Ameen, S., Akhtar, M. S., Seo, H. K. \& Shin, H. S. Advanced ZnO-graphene oxide nanohybrid and its photocatalytic applications. Mater. Lett, 100, 261-265 (2013).

59. Cusco, R. et al. Temperature dependence of raman scattering in ZnO. Phys. Rev. B, 75, 165202 (2007).

\section{Figures}




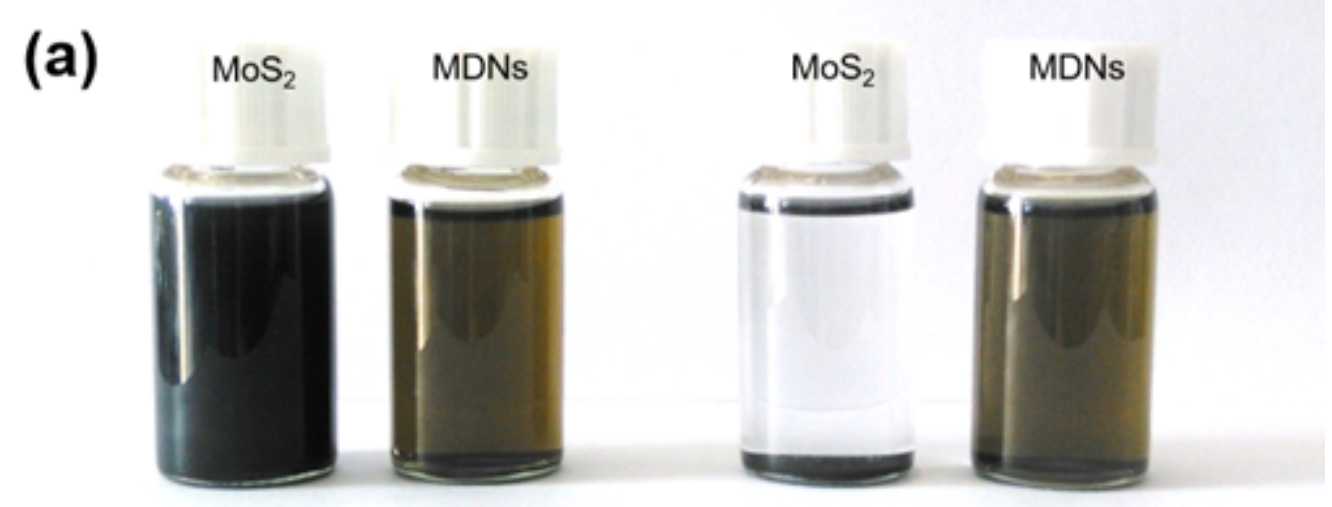

\section{Before storage}

After storage
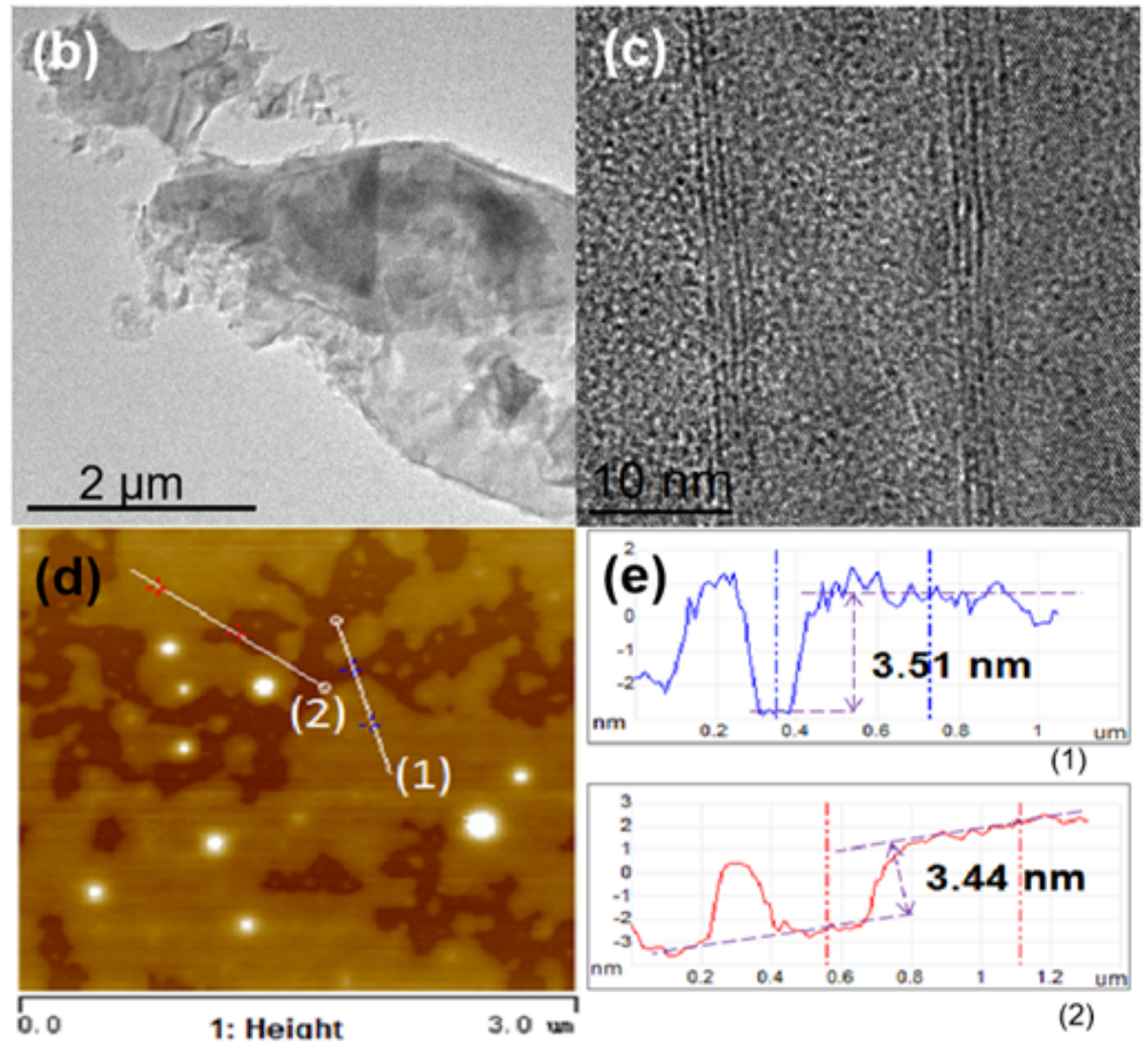

(1)

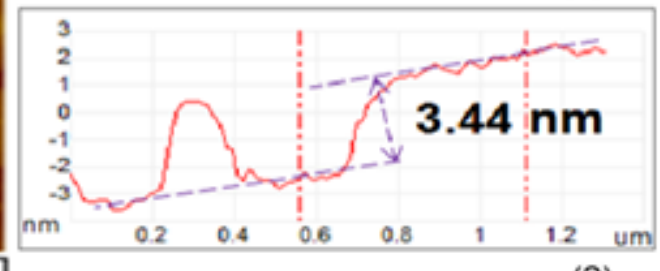

(2)

\section{Figure 1}

(a) Photographs of suspension containing starting MoS2 powders and MDNS in NVP. The suspensions were stored for one week under ambient conditions. (b) TEM image of the prepared MDNS. (c) Highresolution TEM image of the MDNS. (d) AFM image of the MDNS on freshly cleaved mica. (e) Crosssectional analysis along the lines shown in the AFM image. 


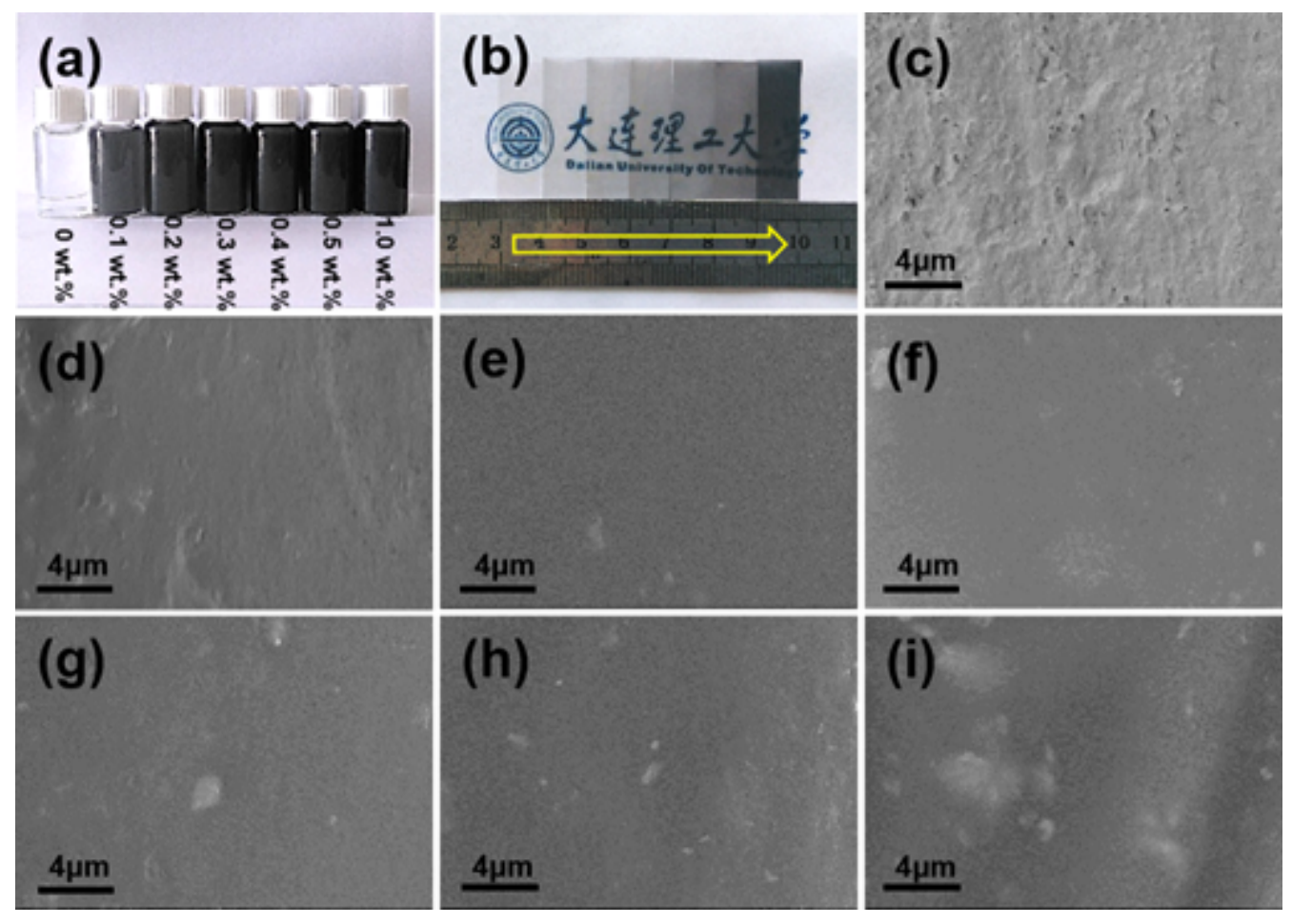

Figure 2

(a) Photograph of prepared paints with different MDNS wt.\% loading after storage for one week. (b) Photograph of free-standing film strips with increasing MDNS loading (from left to right). SEM images of the coating cross-section: (c) MDNS/PVB0, (d) MDNS/PVB0.1, (e) MDNS/PVB0.2, (f) MDNS/PVB0.3, (g) MDNS/PVB0.4, (h) MDNS/PVB0.5 and (i) MDNS/PVB1.0. (Scale bar=4 $\mu \mathrm{m}$ )
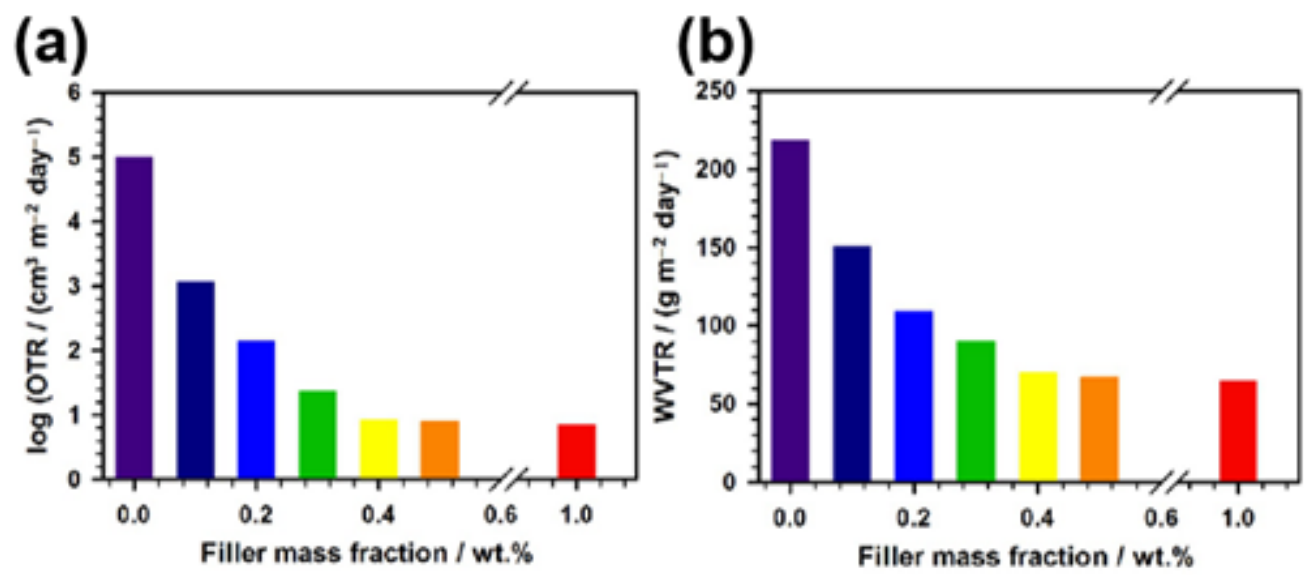

Figure 3

(a) Oxygen and (b) water vapour transmittance rate of MDNS/PVB films with different MDNS loadings. 
(a)

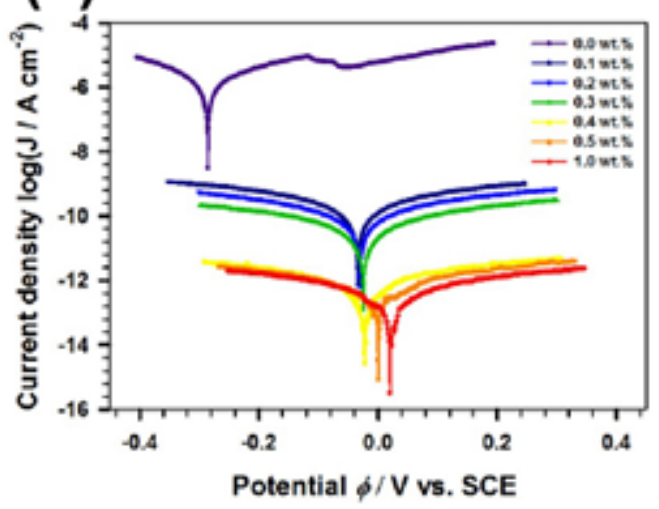

(b)

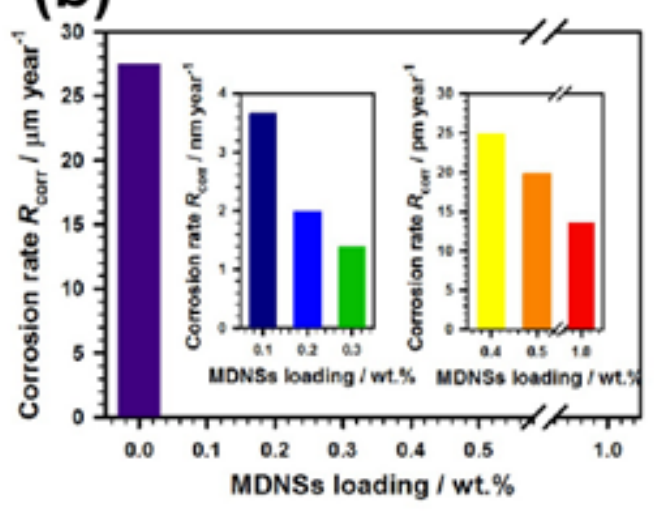

Figure 4

(a) Tafel polarization curves and (b) corrosion rate Rcorr of MDNS/PVB coatings after 4 months of immersion in $3.5 \mathrm{wt}$ \% $\mathrm{NaCl}$ aqueous solution.
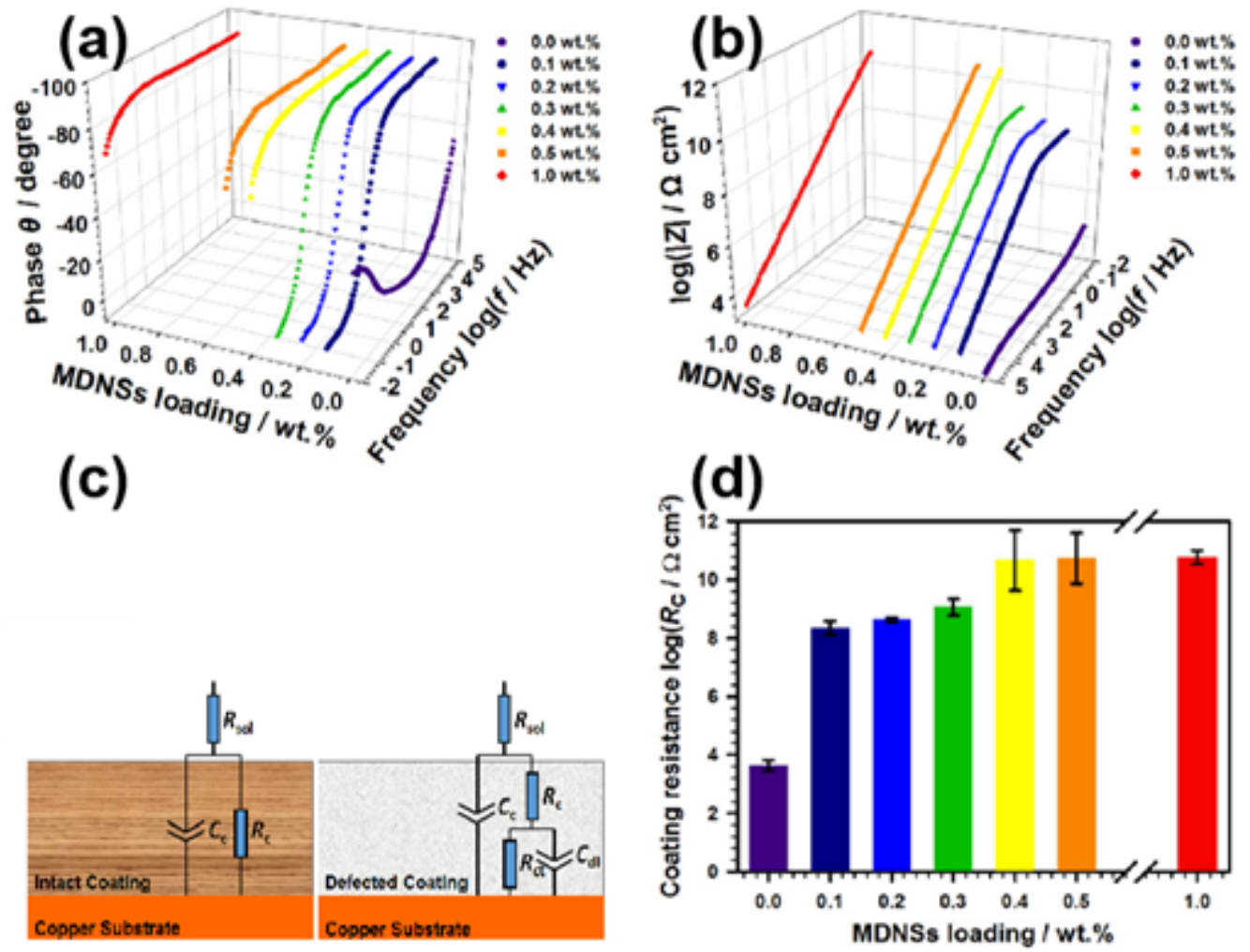

Figure 5

EIS spectra of MDNS/PVB coatings after 4 months of immersion: (a) Bode modulus plots; (b) Bode phase plots; (c) Electrical models of the coatings; (d) Fitting results of the coating resistance Rc. 


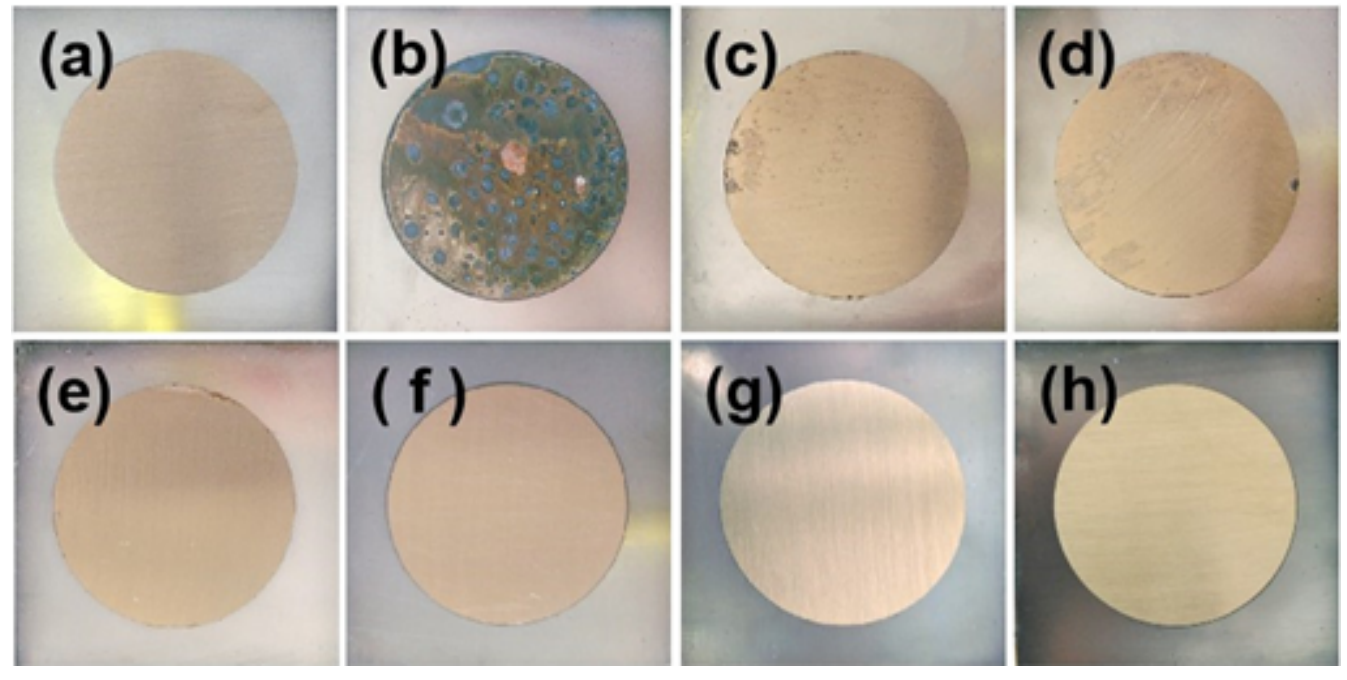

\section{Figure 6}

Photographs of the brass tablets. (a) Typical appearance of newly polished brass tablet. (b-h) Appearances of brass tablets protected by MDNS/PVB coatings for 4 months: (b) MDNS/PVB0, (c) MDNS/PVB0.1, (d) MDNS/PVB0.2, (e) MDNS/PVB0.3, (f) MDNS/PVB0.4, (g) MDNS/PVB0.5 and (h) MDNS/PVB1.0.

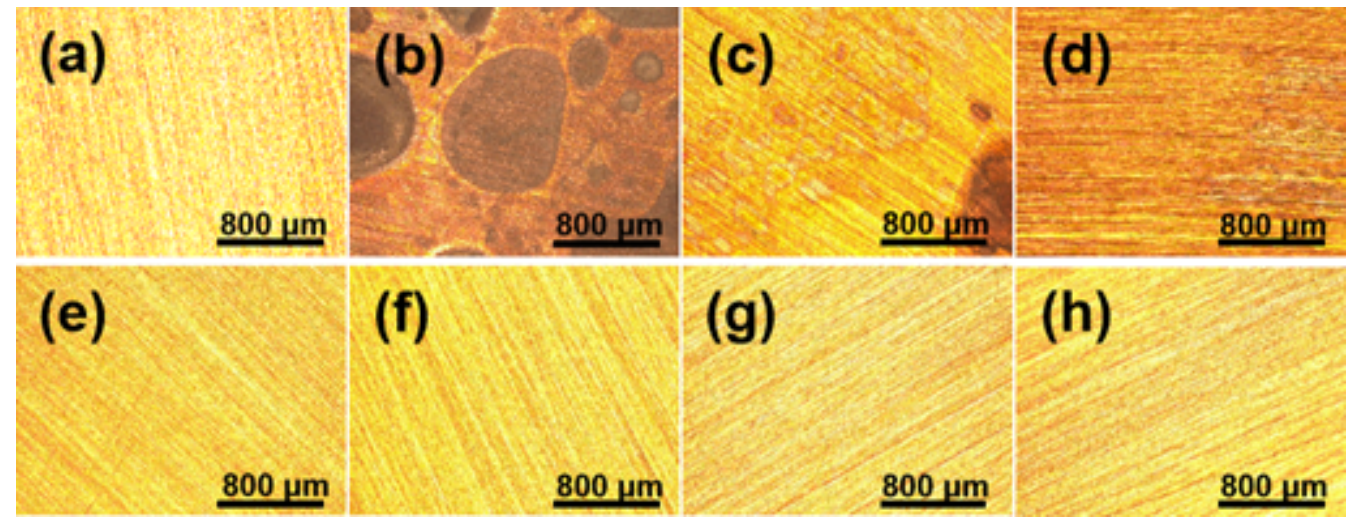

Figure 7

Optical microscope images of the brass tablets. (a) Typical optical microscope image of a newly polished brass tablet. (b-h) Optical microscope images of brass tablets protected by MDNS/PVB coatings for 4 months: (b) MDNS/PVB0, (c) MDNS/PVB0.1, (d) MDNS/PVB0.2, (e) MDNS/PVB0.3, (f) MDNS/PVB0.4, (g) MDNS/PVB0.5 and (h) MDNS/PVB1.0. 


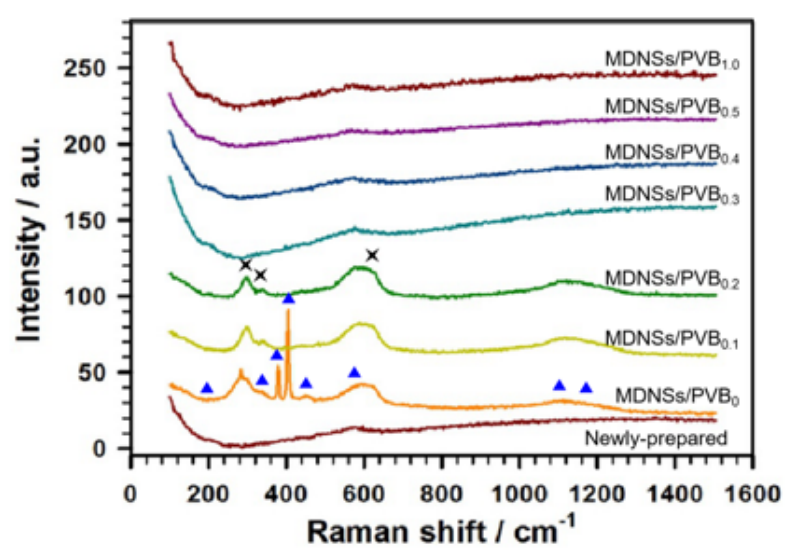

Fig. 8. Raman spectra of corrosion products formed on the brass substrates coated by different coatings after 4 months of immersion in 3.5 wt.\% $\mathrm{NaCl}$ aqueous solution. The symbol $\mathbf{X}$ denotes the Raman activity of $\mathrm{Cu}_{2} \mathrm{O}$, and the symbol $\boldsymbol{\Delta}$ represents the Raman activity of $\mathrm{ZnO}$.

Figure 8

Please See image above for figure legend.

\section{Supplementary Files}

This is a list of supplementary files associated with this preprint. Click to download.

- 9.8SupplementaryMaterial.docx 